\title{
Structural Distress Index: Structural Break Analysis of the Czech and Polish Stock Markets
}

\author{
Michael Princ*
}

\begin{abstract}
:
The estimation of multiple structural break models is usually associated with identification of spurious break points, which are identified by universal algorithms. This leads to overvaluation of structural distress in financial markets represented by data series. The paper is focused on an estimation of the new index, which incorporates results of Student, Bartlett, GLR, Mann-Whitney, Mood, Lepage, Kolmogorov-Smirnov and finally Cramer-von-Mises tests statistics together. The new measure is named Structural Distress Index and evaluates a probability of structural break occurrence based on estimations of proposed models.

SDI values show that Czech and Polish stock markets went through more instable period in 1990s than at the beginning of the global financial crisis in 2007. SDI measure is straightforward and can be easily explained, the highest values of SDI can identify the most important break points of the research period, which starts in year 1993 and ends in year 2014. Universality of SDI offers its further extension and application to further research of financial markets.
\end{abstract}

Key words: Structural break analysis; Stability; Aggregation; Central Europe; Stock markets.

JEL Classification: C13, C51, G14, G15.

\section{Introduction}

In a standard linear econometric approach we assume that a relation between variables is not changing over time. However this approach is usually too simplistic, because in real conditions there are some events, which can influence the substance of economic relations of financial markets, which are manifested in data series. Structural changes can identify whether relationship between dependent and explanatory variables changes over time. When we employ structural change analysis we improve efficiency of estimates, we can switch between different regimes in different time horizons and we can still keep the same linear econometric approach as in Hansen (1992). This offers a great opportunity to use this universal approach of econometric research in many fields. Structural changes and parameter instability is being researched in various scientific disciplines e.g. economics Stock and Watson (1996), finance Andreou and Ghysels (2002), political sciences Piehl et al. (2003) or biostatistics Muggeo (2003). The tests can be very helpful in time of

Michael Princ; Institute of Economics Studies, Charles University, Department of Macroeconomics and Econometrics, Opletalova 26, 11000 Prague, Czech Republic, <mp.princ@seznam.cz>. 


\section{Princ, M.: Structural Distress Index: Structural Break Analysis of the Czech and Polish Stock Markets.}

sudden structural changes. The best example in economics is represented by starting crisis, when a switch from one regime changes to another. Other example can be perceived in opposite event, when crisis phase out and a situation is being stabilized.

We focus our analysis on stock markets in Central European region, namely we compare Warsaw Stock Exchange and Prague Stock Exchange. Stock markets are represented by WIG30 and PX indices. We analyse possibilities of common structural break points, which could have occurred simultaneously on both markets.

While there are many structural break tests it is very difficult to compose their results together. In Princ (2014) it was proved that one of the main problems in the field of applied structural break theory is identification of higher number of break points than we can statistically confirm. Early mentioned challenges were an inspiration for an establishment of a new measure. The new measure is named Structural Distress Index and should offer a new way of aggregation of results and understanding of the internal behaviour of stock markets represented by adequate data series, which should solve mentioned drawbacks and could show the actual tendency of structural distress on financial markets.

\section{Literature Review}

When structural breaks are researched there are two basic meanings changes in structure. There could be either economic point of view explaining a shift in economy represented some important moment e.g. economic crisis, economic integration, or econometric point of view which describes a shift in time series resulting in different coefficients, various volatility levels. The structural change can be understood as either qualitative or quantitative, for more detailed discussion about the topic we recommend Kuan and Hornik (1995).

Structural change in econometric terms can be determined by endogenous change in data sets analysed in many studies including Zivot and Andrews (1992), Perron (1997) or Harvey et al. (2001). When structural break points are analysed we assume that position of break point is unknown as in Andrews (1993).

There are various structural break point tests. We can distinct a class based on identified processes including e.g. sequential probability ratio test in Wald (1945), Cumulated Sum test/control chart (CUSUM) as in Page (1954), recursive CUSUM test of Brown et al.(1975), fluctuation tests for structural changes introduced in Kuan and Hornik (1995), maximum likelihood method of structural break identification established in Hjort and Koning (2002). Other extensive class of structural break point tests is based on computed critical values of various tests statistics for sequences of data series. This class of tests is aimed at identification of changes of predetermined parameters and their results are more straightforward than changes in general framework based on process identification. Other advantage of this class is 
higher number variants, which can be well suited to different conditions. However this advantage results into higher variety in output and possible over-identification.

When we use more methods we arrive to higher number of statistically significant break points. While one method can identify structural break point at one position, other method can just slightly shift the break point. This results into uncertainty, which method we should use. We would like to introduce methodological approach, which will eliminate this uncertainty and moreover utilise all information gained from estimations of various tests. We introduce aggregation of results establishing a new measure describing a propensity of data series to structural break.

While the indicator should display a probability of structural change, which can be understand as a distress in a data series we name the index "Structural Distress Index". Indexation of data structure was used earlier in "Structural SIMilarity Index" (SSIM) developed by Wang et al. (2004), which focused on similarities of data sets representing image output arriving to different conclusions than newly proposed index.

\section{Methodology}

During analysis of time series detecting of structural changes regression relationships has been a focus in various econometric studies. We can distinguish many types of tests on structural changes e.g. Student test, Mood test, MannWhitney test, Lapage test and many other tests devoted to testing of fluctuations. The researched class of structural break tests can be further divided into two main groups. The first group is assuming Gaussian distribution of data series, while the other tests release the assumption. We will discuss two major types of structural change point models, the first class will include statistics testing Gaussian (or normal) sequences of data samples, while the other group will be associated more general framework including Non-Gaussian data sequences, when the distribution of data can be unknown. Thus we distinguish between Gaussian and Non-Gaussian structural break test types, but SDI is not limited to use only these classes and can be further extended as will be further discussed.

\subsection{Gaussian Sequence Models}

In this chapter we will be aiming at parametric and non-parametric change point models including F-statistic, Student-t test and Bartlett test statistic. We would like to start with the most common tests based on traditional F-statistic. F tests are testing null hypothesis of no structural change against an alternative of existence of multiple parameters, which is consistent with Andrews and Ploberger (1994) and Chow (1960). The basic alternative can be defined in a following way as in (1), this idea of instable parameters is also used in other test statistics: 
Princ, M.: Structural Distress Index: Structural Break Analysis of the Czech and Polish Stock Markets.

$$
\beta_{i}=\left\{\begin{array}{l}
\beta_{A}\left(1 \leq \mathrm{i} \leq \mathrm{i}_{0}\right) \\
\beta_{A}\left(\mathrm{i}_{0} \leq \mathrm{i} \leq \mathrm{n}\right)
\end{array},\right.
$$

In our work we employ Student-t test statistic, as in Hawkins et al. (2003), which is used in order to detect mean changes in a Gaussian sequence. And finally we use Bartlett test statistic defined in Snedecor and Cochran (1989) and further improved in Hawkins and Zamba (2005), which is aimed at variance changes detection. The description of Bartlett test statistics is below in equation (2).

$$
X^{2}=\frac{(\mathrm{N}-\mathrm{k}) \ln \left(\mathrm{S}_{p}^{2}\right)-\sum_{i=1}^{k}\left(\mathrm{n}_{i}-1\right) \ln \left(\mathrm{S}_{i}^{2}\right)}{1+\frac{1}{3(\mathrm{k}-1)}\left(\sum_{i=1}^{k}\left(\frac{1}{\mathrm{n}_{i}-1}\right)-\frac{1}{N-k}\right)},
$$

\subsection{Non-Gaussian Sequence Models}

In Non-Gaussian class models we employ Generalized Likelihood Ratio, Mood, Mann-Whitney U, Lepage, Cramer-von-Mises and finally Kolmogorov-Smirnov test statistics.

Generalized Likelihood Ratio is described below:

$$
G L R=k \log \frac{S_{0, n}}{S_{0, \mathrm{k}}}+(\mathrm{n}-\mathrm{k}) \log \frac{S_{0, n}}{S_{\mathrm{k}, \mathrm{n}}},
$$

where $S_{\mathrm{i}, \mathrm{j}}=V_{\mathrm{i}, \mathrm{j}} /(\mathrm{j}-\mathrm{i})$ is defined as a maximum likelihood estimator of the variance of the sequence of data and plugged into (3), for further details we refer to Hawkins and Zamba(2005). The test is used in order to detect possible both mean and variance changes in a Gaussian sequence of data.

The Mood test statistics measures to which extent the rank at each point deviates from its expected value, for further details we refer to Mood (1954). We can define test static in (4) as below:

$$
M=\sum_{x_{i} \in S}\left(r\left(x_{i}\right)-(n+1) / 2\right)^{2}
$$

Mann-Whitney U test proposed in Mann and Whitney (1947), where we compute U test in (5) as a sum of test statistics for two individual samples:

$$
U=U_{1}+U_{2}=R_{1}-\frac{n_{1}\left(\mathrm{n}_{1}+1\right)}{2}+R_{2}-\frac{n_{2}\left(\mathrm{n}_{2}+1\right)}{2},
$$


Lepage test proposed in Lepage (1971), can be defined as a sum of squared MannWhitney and Mood statistics in (6):

$$
L=U^{2}+M^{2},
$$

Cramer-von-Mises test statistics is defined below as a result of maximization process defined in (7).

$$
W_{t}=\max _{k} \frac{W_{k, t}-\mu w_{k, t}}{\sigma w_{k, t}}, 1<k<t
$$

For further details we recommend Anderson (1962) and extension for multiple Ross and Adam (2012).

Kolmogorov-Smirnov test statistics we use is in a form as in Feller (1948) and Ross and Adam (2012), the definition is below in equation (8).

$$
p_{k, t}=Q\left(D_{k, t} \sqrt{\frac{n_{1} n_{2}}{n_{1}+n_{2}}}\right),
$$

For further information about used test statistics and their exact application in parametric and non-parametric we refer to Ross et al. (2011) and Ross and Adam (2012).

\subsection{Structural Distress Index}

High number of different structural test statistics makes it difficult to find out, which structural breaks should be identified as the most important. There are usually two reasons for this improper identification. At first routines using a single test statistics can identify multiple breakpoints, secondly when we use more test statistics it is difficult to distinguish which should be prioritized. Thus this paper is focusing on understanding and combining of results of various structural break tests in order to make a guide through a probability of structural break point occurrence. We would like to offer a new way how to incorporate various results into one and straightforward outcome. This leads to proposal of new "Structural Distress Index" or SDI based on probabilistic approach described in further chapters. SDI is constructed in order to show a probability that a structural break occurs. Our SDI is composing together outputs from Student, Bartlett, GLR, Mann-Whitney, Mood, Lepage, Kolmogorov-Smirnov and finally Cramer-von-Mises tests statistics. Thus we are employing information from 8 individual models together, which would be otherwise very problematic.

While there are many types of models describing change-points or changes in structures of data samples, it is very difficult for researches to perceive outcomes 
Princ, M.: Structural Distress Index: Structural Break Analysis of the Czech and Polish Stock Markets.

from more models in some complex overview. Thus we propose a new way how to utilize output of all previously mentioned using Structural Distress Index (SDI). At first we identify structural change points using each of $n$ models and receive vector, which is constituted from individual structural break points, which are a part of the whole data sample $D$ as in:

$$
V_{i}=\left\{v_{i j}, v_{i j}, \ldots, v_{i j}\right\} ; i \in(1, \ldots, n) ; \mathrm{j} \in(1, \ldots, \mathrm{m}) ; v_{i j} \in D,
$$

While structural break point occur at one point, during construction of SDI we assume that we have $n \times m$ sets of individual components $c_{n m}$ defined as follows:

$$
c_{n m} \mid X_{i j} \sim N\left(v_{i j}, 1\right),
$$

We suppose that $X_{i j}$ is a discrete random variable where:

$$
\sum_{u} \operatorname{Pr}\left(X_{i j}=u\right)=1 \text {, }
$$

SDI is then constructed as a sum of all perceived probability density functions and divided by a correction factor $f_{c}$. In our case we distribute the probability over 5 day horizon. We assume that occurrence is

$$
f_{c}=n \times \max \operatorname{Pr}\left(\mathrm{X}_{i j}=u\right) ; i \in(1, \ldots, n) ; \mathrm{j} \in \mathrm{D},
$$

This definition of correction factor offers that the SDI coefficient stands in an closed interval from 0 to $1, S D I_{t} \in\langle 0 ; 1\rangle$. The principle of SDI computation is similar to CUSUM test statistics as in Ploberger and Kramer (1992), but inclusion of correction factor ensures in computational stability. In our initial phase of research we defined correction factor as equally weighted, but this does not limit its usage for more general cases like geometric mean or others.

The final form of Structural Distress Index is defined at each point of the whole data sample as below:

$$
S D I_{t}=\frac{\sum_{i=1}^{n} \operatorname{Pr}\left(\mathrm{X}_{i t}=t\right)}{f_{c}}, t \in D,
$$

This normalization method also ensures that SDI can be interpreted as a propensity to occurrence of structural break in the data sample. SDI is not correlated with other values and shows additional information about data sample, which can be used in 
further scientific analysis and research of various statistics or models. The definition described above ensures the universality of SDI for further extensions.

\section{Data}

All following data series are captured on a daily basis, which is a suitable application for medium-to-long term strategies.

Observed data samples start from 7th September 1993 and 1 March 2014. This means that a very long history of stock markets is being analysed this includes initial periods after privatization, internet bubble or global financial crisis. Precise descriptive statistics are available in Appendix.

Data estimated in the routine were calculated in a logarithmic form of returns as is described in a relation below (25).

$$
r_{t}=\log \left(p_{t} / p_{t-1}\right) \times 100
$$

where ${ }^{p_{t}}$ stands for closing value of computed index. This means that input values of national stock indices were transformed into daily returns $r_{t}$.

\section{SDI Estimate}

In this chapter we show actual values of Structural Distress Indices for Czech a Polish markets. We will show how we can relate value of SDI with number of breakpoints and we will describe common different features of analysed markets.

Comparison of Figures 1 and 2 shows an interesting result that global financial crisis did not increase SDI or rather a propensity to structural breaks. We can clearly identify that for both markets their initial phase of growth in 1990s was more intensive period of time when structural break $\mathrm{s}$ occurred. The most consistent structural breaks occurred before internet bubble in 1990s and after the global financial crisis in period between 2008 and 2011. 
Princ, M.: Structural Distress Index: Structural Break Analysis of the Czech and Polish Stock Markets.

\section{Fig. 1: SDI for PX index}

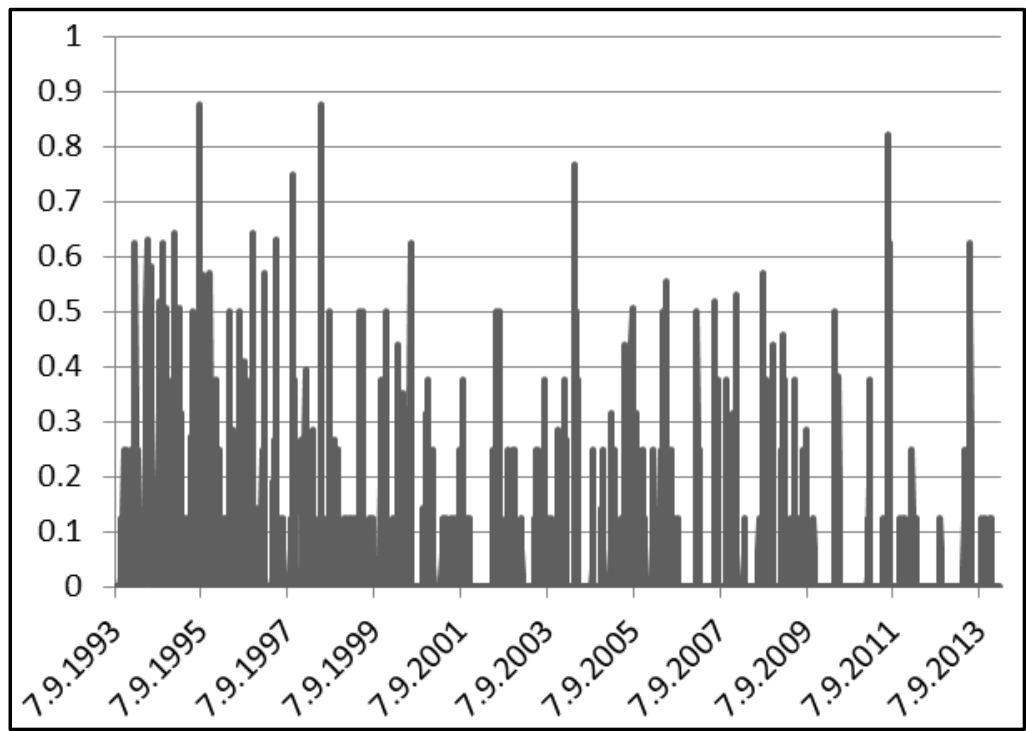

Source: Thomson Reuters, authorial calculation.

\section{Fig. 2: SDI for WIG30 index}

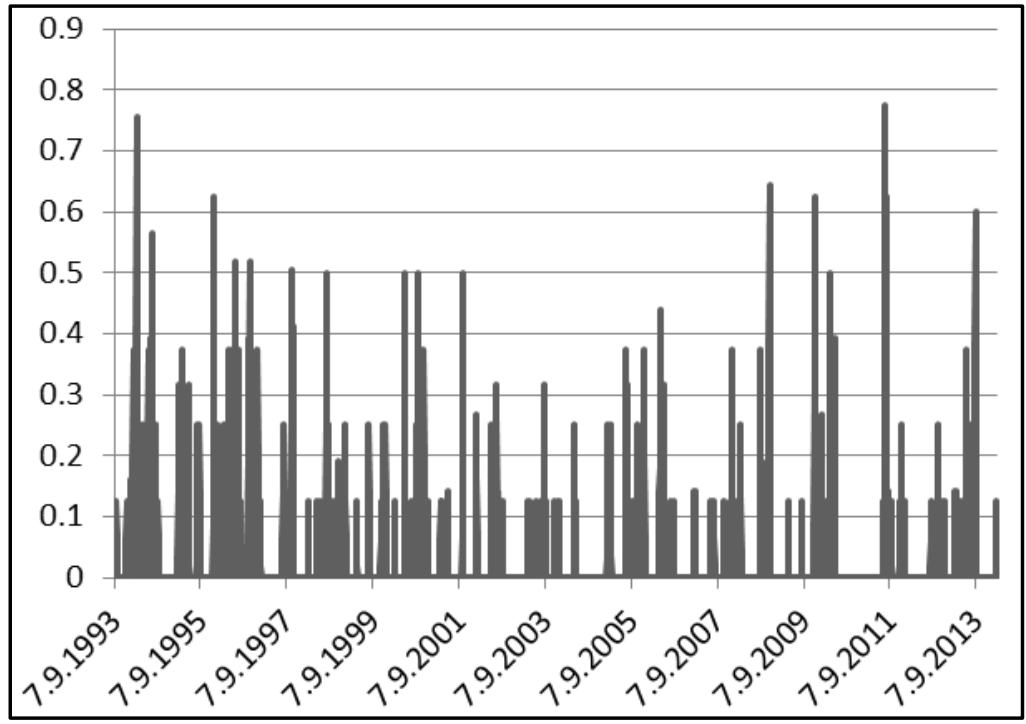

Source: Thomson Reuters, authorial calculation.

We can interpret high SDI values as consistent identification of structural break points, when SDI is over 0.7 it means that cumulated probability from all used structural break point tests is over $70 \%$ for Warsaw Stock Exchange and even over 
$80 \%$ for Prague Stock Exchange. ${ }^{1}$ Exact maximum values are available in Appendix. This result is very desirable, because we can confirm that structural break models are capable of a high consistency. This offers opportunity for further research when number of breakpoints is decreased and loner sub-samples are being analysed. As was shown in Princ (2014) a high number of spurious breakpoints is a very important problem of researched class of models and thus SDI is a very promising remedy to this problem. SDI is capable of an automatic exclusion of nonconsistent breakpoints.

In the next section we will show how we can relate value of SDI with number of possible structural break points. We can confirm the indirect proportion between value of SDI and number of possible break points on Figure 3. It is also interesting that while WIG30 tends to be more instable ${ }^{2}$ on low values of SDI its higher values of SDI propose similar number of breakpoints.

\section{Fig. 3: Relation between Value of SDI and Number of Possible Break Points}

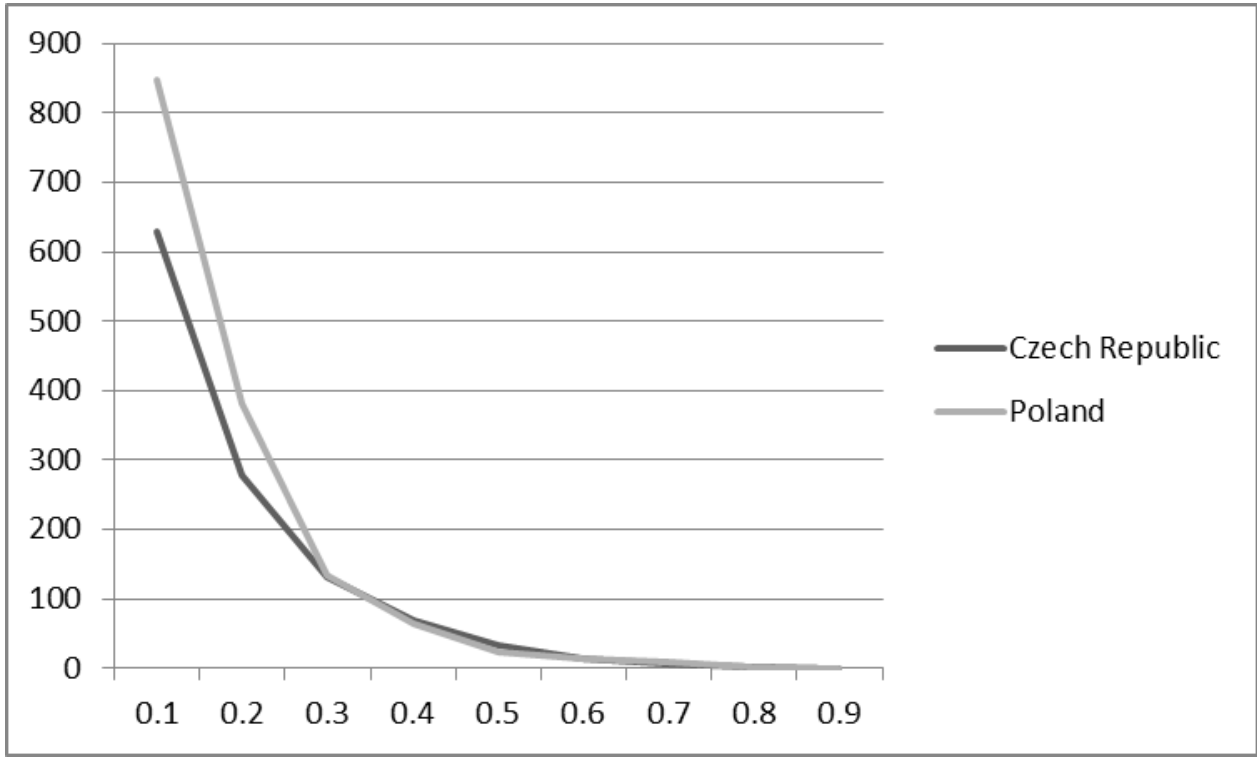

Source: Thomson Reuters, authorial calculation.

More precise description of output is described in the Table 1. We can see that number of breakpoints is steadily decreasing with increasing SDI, which marks a

1 The SDI can be 1 at maximum in a case that all structural break point tests identify the same break point.

2 We refer to stability or instability tendencies based on definition of SDI, which can be characterized as propensity to structural change and thus also a propensity to instability. 
Princ, M.: Structural Distress Index: Structural Break Analysis of the Czech and Polish Stock Markets.

barrier between more or less breakpoints. More detail information about SDI is available in Appendix, which describes positive skewness and kurtosis.

Tab. 1: Relation between Value of SDI and Number of Possible Break Points

\begin{tabular}{lrrrrrrrrr}
\hline SDI & $\mathbf{0 . 1}$ & $\mathbf{0 . 2}$ & $\mathbf{0 . 3}$ & $\mathbf{0 . 4}$ & $\mathbf{0 . 5}$ & $\mathbf{0 . 6}$ & $\mathbf{0 . 7}$ & $\mathbf{0 . 8}$ & $\mathbf{0 . 9}$ \\
\hline PX & 630 & 277 & 132 & 68 & 32 & 15 & 6 & 3 & 0 \\
WIG & 848 & 381 & 135 & 32 & 24 & 13 & 8 & 2 & 0 \\
\hline
\end{tabular}

Source: Thomson Reuters, authorial calculation.

\section{Conclusion}

We have shown that SDI is an effective tool, which can incorporate output from different structural break models in straightforward and clear form. In the initial phase of development we showed that break point occurrence was not increased in the beginning of the global crisis, but rather after its peak in 2008. The interpretation of SDI offers new information about analysed data series and aggregates output of various models into a single output.

Universality of SDI offers further inclusion of more structural break point models. Their role can be adjusted by adjustment of correction factor, which can be represented by geometric mean, median or other measures. In contrast to SSIM by Wang et al. (2004), SDI can perceive changes of internal structure of data series and reveal possible errors of non-robust estimations in advance. We encourage to use SDI for preliminary data analysis, which can reveal some inherent data irregularities and improve consistency of estimations.

We have shown that structural instability was inherent in initial phase of researched stock market in 1990s, when median SDI values were revealed, but SDI also reached its peak values. Preliminary results of SDI can be perceived as a prospect of future research of multi-model estimations.

\section{References}

Anderson, T. W., 1962. On the distribution of the two sample Cramer-von Mises Criterion. Annals of Mathematical Statistics 3, 1148-1159. DOI: 10.1214/aoms/ 1177704477.

Andreou, E., Ghysels E., 2002. Detecting Multiple Breaks in Financial Market Volatility Dynamics. Journal of Applied Econometrics 5, 579-600. DOI: 10.1002/jae.684.

Andrews, D. W. K., 1993. Tests for parameter instability and structural change with unknown changepoint. Econometrica 4, 821-856. DOI: 10.2307/2951764. 
Andrews, D. W. K., Ploberger W., 1994. Optimal tests when a nuisance parameter is present only under the alternative. Econometrica 6, 1383-1414. DOI: 10.2307/ 2951753.

Brown, R. L., Durbin, J., Evans, J. M., 1975. Techniques for testing the constancy of regression relationships over time. Journal of the Royal Statistical Society B 2, 149163. DOI: $10.2307 / 2984889$.

Chow, G. C., 1960. Tests of equality between sets of coefficients in two linear regressions. Econometrica 3, 591-605. DOI: 10.2307/1910133.

Feller, W. E., 1948. On the Kolmogorov-Smirnov Limit Theorems for Empirical Distributions. The Annals of Mathematical Statistics 2, 301-302. DOI: 10.1214/ aoms/1177730243.

Hansen, B. E. 1992. Testing for parameter instability in linear models. Journal of Policy Modeling 4, 517-533. DOI: 10.1016/0161-8938(92)90019-9.

Harvey, D. I., Leybourne, S. J., Newbold, P., 2001. Innovational outlier unit root tests with an endogeneously determined break in level. Oxford Bulletin of Economics and Statistics 5, 559-575. DOI: 10.1111/1468-0084.00235.

Hawkins, D., Qiu, P., Kang, C., 2003. The Changepoint Model for Statistical Process Control. Journal of Quality Technology 4, 355-366.

Hawkins, D., Zamba, K., 2005. Statistical Process Control for Shifts in Mean or Variance Using a Changepoint Formulation. Technometrics 2, 164-173. DOI: 10.1198/004017004000000644.

Hjort, N. L., Koning, A., 2002. Tests for Constancy of Model Parameters Over Time. Nonparametric Statistics 1-2, 113-132. DOI: 10.1080/10485250211394.

Kuan, C. M, Hornik. K., 1995. The generalized fluctuation test: A unifying view. Econometric Reviews 2, 135-161. DOI: 10.1080/07474939508800311.

Lepage, Y., 1971. Combination of Wilcoxians and Ansari-Bradley Statistics. Biometrika 1, 213-217. DOI: 10.2307/2334333.

Mann, H. B., Whitney, D. R., 1947. On a Test of Whether one of Two Random Variables is Stochastically Larger than the Other. Annals of Mathematical Statistics 1, 50-60. DOI: $10.1214 /$ aoms/1177730491.

Mood, A. M., 1954. On the Asymptotic Efficiency of Certain Nonparametric TwoSample Tests. The Annals of Mathematical Statistics 3, 514-522. DOI: 10.1214/aoms/1177728719.

Muggeo, V. M. R., 2003. Estimating Regression Models with Unknown BreakPoints. Statistics in Medicine 19, 3055-3071. DOI: 10.1002/sim.1545.

Page, E. S., 1954. Continuous Inspection Scheme. Biometrika 1/2, 100-115. DOI: 10.1093/biomet/41.1-2.100. 
Princ, M.: Structural Distress Index: Structural Break Analysis of the Czech and Polish Stock Markets.

Perron, P., 1997. Further Evidence on Breaking Trend Functions in Macroeconomic Variable. Journal of Econometrics 2, 355-385. DOI: 10.1016/s0304-4076(97)000493.

Piehl, A. M., Cooper, S. J., Braga, A. A., Kennedy, D. M., 2003. Testing for Structural Breaks in the Evaluation of Programs. Review of Economics and Statistics 3, 550-558. DOI: 10.1162/003465303322369713.

Ploberger, W., Kramer, W., 1992. The CUSUM test with OLS residuals. Econometrica 2, 271-285. DOI: 10.2307/2951597.

Princ, M., 2014. Testing Gaussian and Non-Gaussian Break Point Models: V4 Stock Markets, Optimization, Education and Data Mining in Science. Engineering and Risk Management Working Papers vol. 8.

Ross, G. J., Adams, N. M., 2012. Two Nonparametric Control Charts for Detecting Arbitary Distribution Changes. Journal of Quality Technology 2, 102-116.

Ross, G. J., Tasoulis, D. K., Adams, N. M., 2011. Nonparametric Monitoring of Data Streams for Changes in Location and Scale. Technometrics 4, 379-389. DOI: 10.1198/TECH.2011.10069.

Snedecor, G. W., Cochran, W. G., 1989. Statistical Methods. Iowa State University Press.

Stock, J. H., Watson, M. W., 1996. Evidence on Structural Instability in Macroeconomic Time Series Relations. Journal of Business \& Economic Statistics 1, 11-30. DOI: 10.2307/1392096.

Wald, A., 1945. Sequential Tests of Statistical Hypotheses. Annals of Mathematical Statistics 2, 117-186. DOI: 10.1214/aoms/1177731118.

Wang, Z., Bovik, A. C., Sheikh, H. R., Simoncelli, E. P., 2004. Image quality assessment: From error visibility to structural similarity. IEEE Transactions on Image Processing 4, 600-612. DOI: 10.1109/tip.2003.819861.

Zivot, E., Andrews, D. W. K., 1992. Further Evidence on the Great Crash, the OilPrice Shock, and the Unit-Root Hypothesis. Journal of Business \& Economic Statistics 3, 251-270. DOI: 10.1080/07350015.1992.10509904. 
European Financial and Accounting Journal, 2016, vol.11, no. 3, pp. 125-137.

\section{Appendix:}

\section{Data Statistics}

\begin{tabular}{lrrrrrrrrr}
\hline & Mean & \multicolumn{1}{l}{ SD } & Media & MAD & \multicolumn{1}{c}{ Min } & Max & Skewness & Kurtosis & \multicolumn{1}{c}{ SE } \\
\hline PX & 852.02 & 411.83 & 780.2 & 457.31 & 316 & 1936.1 & 0.7 & -0.58 & 5.84 \\
WIG & 1925.6 & 727.02 & 1797.3 & 794.05 & 577.8 & 3935.5 & 0.47 & -0.35 & 10.25 \\
SDI PX & 0.04 & 0.09 & 0 & 0 & 0 & 0.88 & 3.75 & 17.29 & 0 \\
SDI WIG & 0.02 & 0.07 & 0 & 0 & 0 & 0.77 & 4.9 & 30.9 & 0 \\
\hline
\end{tabular}

Source: Thomson Reuters, authorial calculation.

Note: SD - Standard Deviation; MAD - Median Absolute Deviation; SE - Standard Error. 
